\title{
Anxiety and atrial fibrillation: an interesting bidirectional association.
}

\author{
Ashraf Alqaqa* \\ Department of Cardiology, Tennova Healthcare, Tennessee, United States of America
}

\begin{abstract}
Atrial fibrillation stands as one of the most common supraventricular arrhythmias. Its incidence in general population has escalated significantly over the last few decades. The health-related quality of life (HRQoL) of patients with atrial fibrillation is currently a vital concern in cardiovascular health management. Accordingly, key elements of meager HRQoL of AF patients demand better clarification. Nowadays, several reports have tested the mutual relation between anxiety and AF initiation and progression. Significantly, anxiety affects how the patients approach their disease and, additionally, anxiety may affect the efficiency of various AF therapies. The goal of this research is to explore an area that considered by patients to be challenging before the therapeutic work-up. Study concluded that there is a multifaceted relationship between AF and anxiety. AF can ground anxiety among patients and equally, anxiety can pave a background that is favorable for the initiation and Progression of AF.
\end{abstract}

Keywords: Atrial fibrillation; Anxiety; Cardiovascular disease; Treatment; Epidemiology.

Accepted on January 12, 2017

\section{Introduction}

Atrial fibrillation (AF) is the most widely recognized cardiovascular arrhythmia. Diabetes, male gender, congestive heart failure, obesity, and hypertension are major risk factors contributing to the increasing prevalence of AF. Furthermore, $\mathrm{AF}$, associated with augmented morbidity and mortality rates, has been correlated with an inferior quality of life and amplified risk of cardiac failure and cerebrovascular accidents [1].

While several studies have evaluated the passive influence of anxiety, and other psychological disturbances, on health related outcomes of variable cardiac disorders, the mutual effect between AF and anxiety remains elusive. While psychological comorbidities may have an impact of AF manifestation, the disease course affects the personal satisfaction and the quality of life. The explanation and directionality of such correlation warrant further assessment. Subsequently, this evaluation will be reflected, eventually, in better patient outcomes.

Accordingly, the aim of the current review is to: (1) studying the prevalence of anxiety-associated AF through the appraisal of the related literature (2) evaluation of the distinctive effect of anxiety in AF patients (3) ascertain an evidence of pathophysiological crosstalk between anxiety and AF (4) elucidate the effect of anxiety, if any, on management of AF.

\section{Methods}

Different databases "Embase, EconLit, Google Scholar, Medline, PubMed, ProQuest, Scopus, Springer Link and Science Direct" were subjected to a comprehensive literature mining in the period from 2001 till December 2016.

In various combinations, "Atrial Fibrillation", "Anxiety", "Cardiovascular disease", "Treatment" and "Epidemiology" were used as keywords using BOOLEAN and MeSH search.
Additional publications, reached through a manual search of references of related papers and review articles, were also cited when applicable.

\section{Results and Discussion}

Primarily, 213 studies were retrieved through the abovementioned searching method. Such list was shortened to 152 as 62 were duplicates and therefore omitted. Out of enduring articles, 70 were excluded based on irrelevant title and/or abstract. Subsequently, full text of 81 articles were appraised and 48 studies were further disqualified as these failed to provide relevant details. Accordingly, 33 articles were particularly selected for this review.

\section{Anxiety in AF patients}

Numerous reports analyzing the anxiety among cardiac patients are currently carried out in coronary heart disorder patients [2]. It has been stated that patients suffering AF only are subjected to an increased rate of psychological distress. Thrall et al. reported that about $28 \%$ and $38 \%$ of AF patients were supposed to have state and trait anxiety according to State Trait Anxiety Inventory (STAI). Whereas, the height of trait anxiety was significantly higher compared to those patients bearing other chronic disorder, hypertension as an example, assessed along the study $(38 \%$ among AF patients vs. $22 \%$ among hypertensive patients, $=0.03$ ) [3] .

In addition, height of anxiety is correlated with quality of life among AF patients [3]. Moreover, the mortality risk was almost one third higher among men with $\mathrm{AF}$ and suffering depression compared to men without depression. The anxiety among AF men compared to AF women patients, no associations were found [1]. Furthermore, levels of anxiety among AF patients was not significantly changed along six months of follow up. Because of this disease limitations, satisfaction rates among AF patients were found to be significantly decreased as apparent in 
their work, activities, social as well as sexual life and spare time $[4,5]$. Interestingly, Dabrowski et al. indicated that women with AF, distinctively, suffer depression and sleep disturbances as compared to men with AF [4]. In addition Ong et al. reported a meager quality of life among AF females compared to male's patients [6]. Indicated in AF-CHF trial of rate versus rhythm control strategies, Frasure-Smith et al. stated anxiety as a cause of prolonged cardiovascular morbidity among AF patients and heart failure [7].

Remarkably, Perret-Guillaume et al. reported that physical manifestations of $\mathrm{AF}$ are found to bear less clinical consequence than psychological. In order to provide proofs, data have been aggregated from elderly populations through Duke Health Profile which returned major discrepancy in mental function and anxiety in AF patients compared to control. On the contrary, the comparison revealed no statistically major differences in measures of physical health, social impairment, or disability [8].

On the other hand, in a study hypothesizing a difference between Permanent AF patients and normal sinus rhythm population in terms of anxiety, depression, and sleep impairment, it was found that there is no significant difference of anxiety among AF patients versus control. Compared to subjects in sinus rhythm, AF patients had significantly poorer scores for SF-36 physical and social functioning. Consequently, it could be noted that physical manifestations are accounting for diminishing the quality of life in AF patients. However, the incongruent findings could be attributed to the fact that authors select only those patients having lasting and clinically constant AF [9].

\section{Pathophysiological link between $\boldsymbol{A F}$ and anxiety}

Various studies have emphasized the augmented anxiety rate among AF patients attributed to the poor quality of life. Paradoxically, little information is known about the possibility of triggering AF by anxiety. In a study by Eaker et al. it was obvious that anxiety could be one of the predictors of ten-year incidence of AF in both male and females [10].

Inflammation and oxidative stress are supposed to be key players in development and stimulation of AF [11]. In relation, Patients with anxiety express enhanced levels of acute phase reactants i.e. C-reactive proteins, pro-inflammatory cytokines, and decreased levels of anti-inflammatory molecules [12]. Remarkably, Rommel et al. reported a higher hs-CRP levels in anxiety-associated in contrast to no or minimal anxiety in an AF suffering population [13]. In relation, Son and Song reported augmented hs-CRP levels in AF patients possessing "Type D" personality compared to those with non-type-D personality [14]. Significantly, the findings of Son and Song and Tully et al. however, were established in those patients who previously have AF $[14,15]$.

On the other hand, anxiety patients are more likely to have increased activity of sympathetic nervous system [16]. Hansson et al. suggested that catecholamine release due to the psychic stress is a provocative factor among paroxysmal AF patients. However, one of the pitfalls of this study is being only based on the patient's views about what initiate their AF while no psychiatric measures were implemented [17]. In congruence, after cardiac surgery, anxiety manifestations augmented the risk of AF, as reported by Tully et al. In sum, increased sympathetic tone, lessened vagal tone, and the cardinal symptoms of anxiety, could be major provocative factors of postoperative AF [15].

Additionally, those individual who are suffering from anxiety have a stimulated hypothalamic-pituitary-adrenal axis and renin-angiotensin-aldosterone system [18]. In relation, cardiac fibrosis could be induced by the high levels of angiotensin II, which activate mitogen activated protein kinases and diminish collagenase activity. Moreover, binding of angiotensin II to angiotensin II type I receptors induces growth factor (TGF)-1 production, a trigger for atrial fibrosis [19]. In brief, despite the absence of solid evidence to prove that anxiety activate new onset $\mathrm{AF}$, it could be extrapolated that the comorbidity provides a background that is favourable of the initiation and prolongation of AF.

\section{The effect of AF treatment on anxiety and impact of anxiety on treatment success}

Strategies implemented to treat AF comprise electrical cardio version, catheter ablation and pharmacological agents. The report that is made by AF-CHF trial of rate versus rhythm control, reported no benefit found among rhythm versus rate control, in prevention of death among those patients enduring symptoms of anxiety [7]. Additionally, Frasure-Smith et al. reported lower cardiovascular mortality in rhythm control group in those patients who have $\mathrm{CHF}$ and $\mathrm{AF}$ with greater sensitivity to anxiety compared to those that receiving rate control [20].

Quality of life can be enhanced through the restoration of sinus rhythm using electrical cardio version [21]. The influence of electrical cardio version on anxiety-associated AF patients is still questioned similar to pharmacologic rate and rhythm strategies. However, Lange and Herrmann-Lingen found that after successful electrical cardio version, risk of recurrence of AF remains existent due to the anxiety. For those AF patients who scored more than 7 on HADS, $85 \%$ have the possibility of recurrence. Moreover, Yu et al. reported an increased risk of AF recurrence due to anxiety after taking circumferential pulmonary vein ablation [22]. Type D personality, anxiety score of HADS, elevated adrenergic tone and proinflammatory mediators are known to promote the recurrence rates [23].

As several studies exhibited remarkable improvement among those anxiety patients who underwent catheter ablation [22,24], various patients who have undergone catheter ablation were assessed for long and short term benefit. To assess the quality of life, 7 generic and specific tools are used. Remarkably, quality of life was improved after the short and long term follow up among patients with paroxysmal and constant AF through the use of 7 tools devoid of considering the success of catheter ablation [25]. Furthermore, more competence in Hospital Anxiety scale score was found after passing a successful ablation [24]. Likewise, Wokhlu et al. stated that ablation efficacy is not the sole factor responsible for 
improving quality of life. In fact, additional factors as baseline quality of life, discontinuation of anticoagulation drugs, and symptom relief are also important as well [26].

Moreover, Fichtner et al. reported better results in disease specific questionnaire i.e. severity of AF scale and checklist of AF symptom, among those patients who are taking long term follow up as compared to those patients who experienced a failed ablation [25]. In both of the studies efficacy in quality of life is because of short term results of placebo effect but it can't be spread on to get long term benefit. Patients felt much better as they were in state of sinus rhythm as the AF burden is being released and no medicines were used.

Turkey et al. reported that dabigatran-treated AF had lower HAS score compared to warfarin treated. It could be extrapolated from this study that dabigatran may ameliorate the HQoL and decrease morbidity and morbidity due to the reduction of anxiety levels [5].

Current information regarding the availability and benefit of alternative medicines in managing anxiety-associated $\mathrm{AF}$ is scarce. Yoga training reduced symptomatic and asymptomatic AF episodes, depression and anxiety. In addition, it improved the QoL parameters including social functioning, and mental health. Moreover, heart rate and systolic and diastolic blood pressure exhibit significant difference before and after yoga [27]. In paroxysmal AF patients, yoga exercise could be integrated as a low cost alternative to conventional management in AF and anxiety handling.

\section{Conclusion}

This article represents a proof of concept that a relationship exists between anxiety and AF. The latter can ground the former among patients and equally, the former can pave a background that is favourable for the initiation and Progression of the latter.

Considerably, anxiety affects how patients, especially in women, approach their disease. The incidence of anxiety may affect the efficiency of various AF therapies. Hence, executing strategies reducing anxiety in AF patients could improve treatment consequences, patients HRQOL, and lower financial difficulties related to AF. These strategies comprise; patient orientation about disease course progression, strict management of AF symptoms, encouraging catheter ablation after antiarrhythmic drugs failure, and possibly treating the patients with anxiolytics. Additional trials are essential to assess the benefits of SSRI practice in decreasing the incidence of AF and HRQOL enhancement in AF patients.

\section{References}

1. Wändell P, Carlsson AC, Gasevic D, et al. Depression or anxiety and all-cause mortality in adults with atrial fibrillation--A cohort study in Swedish primary care. Ann Med. 2016; 48(1-2): 59-66.

2. Nørgaard MW, Pedersen PU, Bjerrum $M$, et al. Visualisation during ablation of atrial fibrillationstimulating the patient's own resources: Patients' experiences in relation to pain and anxiety during an intervention of visualisation. Eur J Cardiovasc Nurs. 2014; 14(6): 552-9.

3. Thrall G, Lip GY, Carroll D, Lane D. Depression, anxiety, and quality of life in patients with atrial fibrillation. Chest. 2007; 132(4): 1259-64.

4. Dąbrowski R, Smolis-Bąk E, Kowalik I, et al. Quality of life and depression in patients with different patterns of atrial fibrillation. Kardiol Pol. 2010; 68(10): 1133-39.

5. Turker Y, Ekinozu I, Aytekin S, et al. Comparison of changes in anxiety and depression level between dabigatran and warfarin use in patients with atrial fibrillation. Clin Appl Thromb Hemost. 2015;23(2): 164-7.

6. Ong L, Irvine J, Nolan R, et al. Gender differences and quality of life in atrial fibrillation: the mediating role of depression. J Psychosom. 2006; 61(6): 769-74.

7. Frasure-Smith N, Lespérance F, Habra M, et al. Elevated depression symptoms predict long-term cardiovascular mortality in patients with atrial fibrillation and heart failure. Circulation. 2009;120(2): 134-140.

8. Perret-Guillaume C, Briancon S, Wahl D, et al. Quality of Life in elderly inpatients with atrial fibrillation as compared with controlled subjects. J Nutr Health Aging. 2010;14(2): 161-6.

9. Ariansen I, Dammen T, Abdelnoor M, et al. Mental health and sleep in permanent atrial fibrillation patients from the general population. Clin. Cardiol. 2011;34(35): 327-31.

10. Eaker ED, Sullivan LM, Kelly-Hayes M, et al. Tension and anxiety and the prediction of the 10-year incidence of coronary heart disease, atrial fibrillation, and total mortality: the Framingham Offspring Study. Psychosom Med. 2005;67(5): 692-6.

11. Dewland TA, Vittinghoff E, Harris TB, et al. Inflammation as a Mediator of the Association Between Race and Atrial Fibrillation: Results From the Health ABC Study (Health, Aging, and Body Composition). JACC Clin Electrophysiol. 2015;1(4): 248-55.

12. Brouwers C, Kupper N, Pelle AJ, et al. Depressive symptoms in outpatients with heart failure: importance of inflammatory biomarkers, disease severity and personality. Psychol Health. 2014;29(5): 564-82.

13. Rommel J, Simpson R, Mounsey JP, et al. Effect of body mass index, physical activity, depression, and educational attainment on high-sensitivity C-reactive protein in patients with atrial fibrillation. Am J Cardiol. 2013;111(2): 208-12.

14. Son YJ, Song EK. The impact of type D personality and high-sensitivity C-reactive protein on health-related quality of life in patients with atrial fibrillation. Eur $\mathbf{J}$ Cardiovasc Nurs. 2012;11(3): 304-12.

15. Tully PJ, Bennetts JS, Baker RA, et al. Anxiety, depression, and stress as risk factors for atrial fibrillation after cardiac surgery. Heart Lung. 2011;40(1): 4-11.

16. Carney RM, Freedland KE, Veith RC, et al. Depression, the autonomic nervous system, and coronary heart disease. Psychosom Med. 2005;67(1): S29-S33. 
17. Hansson A, Madsen-Härdig B, Olsson SB, et al. Arrhythmia-provoking factors and symptoms at the onset of paroxysmal atrial fibrillation: a study based on interviews with 100 patients seeking hospital assistance. BMC Cardiovasc Disord. 2004;3(4): 13.

18. Murck H, Held $\mathrm{K}$, Ziegenbein $M$, et al. The reninangiotensin-aldosterone system in patients with depression compared to controls-a sleep endocrine study. BMC psychiatry. 2003;3: 15 .

19. Takahashi N, Kume O, Wakisaka O, et al. Novel strategy to prevent atrial fibrosis and fibrillation. Circ J. 2012;76(10): 2318-26.

20. Frasure-Smith N, Lespérance F, Talajic M, et al. Anxiety Sensitivity Moderates Prognostic Importance of Rhythmversus Rate-Control Strategies in Patients with Atrial Fibrillation and Congestive Heart Failure: Insights from the AF-CHF Trial. Circ Heart Fail. 2012;5(3): 322-30.

21. Berry C, Stewart S, Payne EM, et al. Electrical cardioversion for atrial fibrillation: outcomes in 'reallife'clinical practice. Int J Cardiol. 2001;81(1): 29-35.

22. $\mathrm{Yu} \mathrm{SB}, \mathrm{Hu} \mathrm{W}, \mathrm{Zhao} \mathrm{QY}$, et al. Effect of anxiety and depression on the recurrence of persistent atrial fibrillation after circumferential pulmonary vein ablation. Chin Med J. 2012;125: 4368-372.

23. Lange HW, Herrmann-Lingen C. Depressive symptoms predict recurrence of atrial fibrillation after cardioversion. J Psychosom Res. 2007; 63(5): 509-513.
24. Mohanty S, Mohanty P, Di Biase L, et al. Influence of body mass index on quality of life in atrial fibrillation patients undergoing catheter ablation. Heart Rhythm. 2011;8: 1847-52.

25. Fichtner S, Deisenhofer I, Kindsmüller $\mathrm{S}$, et al. Prospective Assessment of Shortand LongTerm Quality of Life After Ablation for Atrial Fibrillation. J Cardiovasc Electrophysiol. 2012;23(2): 121-7.

26. Wokhlu A, Monahan KH, Hodge DO, et al. Long-term quality of life after ablation of atrial fibrillation: the impact of recurrence, symptom relief, and placebo effect. J Am Coll Cardiol. 2010;55(21): 2308-2316.

27. Lakkireddy D, Atkins D, Pillarisetti J, et al. Effect of yoga on arrhythmia burden, anxiety, depression, and quality of life in paroxysmal atrial fibrillation: the YOGA My Heart Study. J Am Coll Cardiol. 2010; 61(11): 1177-1182.

\section{*Correspondence to}
Ashraf Alqaqa
Department of Cardiology
Tennova Healthcare
United States of America 